\title{
An Analysis of How Interactive Technology Supports the Appreciation of Traditional Chinese Puppetry: A Review of Case Studies
}

\author{
Shichao Zhao \\ Newcastle University, Newcastle, NE4 5TG, UK \\ s.zhaollenewcastle.ac.uk
}

\begin{abstract}
From the perspective of safeguarding Chinese Cultural Heritage, this paper discusses how to enhance the appreciation of traditional Chinese puppetry through the support of interactive technology. The author analyses extensive, yet current case studies, based on the findings described in the interactive systems for puppetry performances and interactive technology for puppetry appreciation. The author summarises four aspects of how to enhance the appreciation of, and engagement with, traditional Chinese puppetry: (1) maintaining originality is necessary for the design phase; (2) it is crucial to explore how to use interactive technology in order to design a way for adults to appreciate this form of art; (3) it is also necessary to determine ways to support adult audiences in grasping the cultural significance and folk customs of traditional Chinese puppetry; and (4) the study's further main research goals are to investigate ways to use emotional expressions, digital storytelling and other methods in conjunction with interactive technology to help multi-cultural users comprehend traditional Chinese puppetry.
\end{abstract}

Keywords: Traditional Chinese Puppetry, Interactive Technology, Digital Storytelling, Emotional Expression, Cultural Appreciation.

\section{The Dilemmas Facing Traditional Chinese Puppetry}

Puppetry is one of the most significant components of Chinese opera. Like other traditional art forms, the opera has been passed down through face-to-face teaching and incomplete text sources. Furthermore, many puppetry classics are only safeguarded in the memories of elderly folk artists; therefore, these techniques and knowledge are in danger of fading [1, 2]. After 1949, the formats of Chinese puppetry became richer [3]. Apart from preserving traditional opera, puppetry began to mix with other media and began to appear in contemporary plays, TV series and movies. This not only maintained the historical element of puppetry, but also expanded its platform to new audiences. However, just like other Chinese folk-art forms, puppetry is going through a type of culture shock as it encounters a variety of entertainment channels and different target audiences [4]. For example, take the famous marionettes from the city of Quanzhou in Fujian Province; there, the government failed to pay enough attention to the safeguarding of it, and traditional puppetry has since come under threat [3]. These days, 
Quanzhou puppetry can only be seen at festivals or sacrificial ceremonies. Consequentially, less money is invested in the support of professional puppeteers and artists; some have had to abandon their careers, making their art form a dwindling branch of Chinese puppetry.

ICH (Intangible Cultural Heritage) like traditional Chinse puppetry, is only passed down through oral teachings and incomplete writings, making recording and conservation more complex. A plethora of classical puppet shows are only maintained in the memories of practitioners, rendering these skills and knowledge on the verge of extinction [5]. Historically, words, photographs and video recordings have been the most frequently used media for conservation. Although photography and videography have begun to improve the quality of preserving traditional puppetry and other types of ICH, these approaches do not cover every aspect of conservation. Moreover, it is difficult for traditional media to attract audiences from different cultural backgrounds. Previous research on individual puppeteers and other institutions shows that many photographs or audio or video tape scripts have decayed due to their storage environment, as well as climate change since the 1980s. Undoubtedly, this represents a huge loss for Chinese ICH [6].

Moreover, technology could facilitate its cultural safeguarding. Interactive technologies have vast potential to help safeguard ICH, not only in China, but also on an international scale [7-12]. Researchers have used them to represent cultural artefacts; this has often involved digital augmentation to support audience involvement. An important outcome of digitisation is a greater potential to promote cultural artefacts among audiences, as well as the creation of new forms of cultural interactivity. Following this radical design evolution, many interactive projects have been developed to disseminate the objectives of traditional societies [13-16]. Nonetheless, limited attention has been paid to actual, in-depth interpretations and engagement regarding the aesthetics of these artefacts [17]. Carefully applying interactive technologies heightens the potential of enhancing artistic appreciation and the delivery of cultural significance. However, further exploration is needed to understand how to best support this possibility in terms of Chinese ICH [18]. The development of digital techniques has made the conservation of traditional Chinese puppetry more convenient (e.g. the use of digital video cameras or mobile devices to record puppetry). New databases have provided better environments for data recovery and storage. All of these technologies have helped to spread traditional Chinese puppetry [19]. However, none of them offer ways to edit or utilise digital content for audience interactions; thus, the content remains invisible to the general public. Linear video recordings also limit the possibility of further interactive digital setups. Combining interactive digital media and traditional puppetry could improve the recording, teaching, editing, development and performance of the latter. 


\section{Interactive Technology in Traditional Chinese Puppetry}

This section investigates related research on using interactive technology to enhance puppetry performances, and on appreciation in order to derive insight into design in the future case studies.

\subsection{Methods}

Since the 1980s, digital heritage studies have become an independent research area [20]. Text analysis and relevant archival research are frequently used techniques to grasp the essence of past occurrences and to trace the changing meanings of heritage [21]. Specifically, the author adopts three steps which include (1) evaluating other studies; (2) selecting the studies to include in the review; and (3) organising the review. In the first step, the author mainly evaluates the extensive case studies which are using interactive technology to experience the traditional Chinese puppetry, as well as comments on each study's value and validity. In the second step, the author chooses studies which are most relevant and most important for the support of the appreciation and engagement of traditional Chinese puppetry. In the third step, the author organises the different themes based on the different interactive technology to explore the potential design opportunities and insights.

\subsection{Interactive Systems for Puppetry Performances}

This section delves into some case studies on using interactive technology to support puppetry performances. As early as 1998, motion capture systems were devised to transfer puppetry gestures into a digital/virtual form [22]. This created more possibilities for using digital puppetry gestures to aid puppetry performances. Shin and colleagues explored computer puppetry by capturing gestures and fully visualising them in real time. Thus, tools used to create animation extended the availability of puppetry gestures in the entertainment industry, such as performances broadcasted on television [23].

A number of interactive systems have been developed that enable users to create digital puppets and puppetry roles. For instance, as early as 2002, PUPPET, an autonomous agent, populated virtual environments to support children in experiencing and exploring the different roles in a puppetry performance. This system lets children interact in a virtual environment to understand the narrative and characters of a performance. The virtual environment has effectively allowed them to physically interact with the characters [24]. Unlike Marshall and colleagues, Cutout Animation adopted a videobased paper tracking technology to create cut-out-style animations, rather than puppetry roles [25]. Users could make physical puppets by cutting them out of paper; their movements were captured by an overhead camera and rendered into animation on the screen, with the option to choose from different backgrounds. In 2011, a digital system called Shadow Story integrated digital puppet making and puppetry roles, providing children with an interface that lets them employ their own gestures to perform stories [26]. They could also use a pen and tablet input to create digital shadow puppetry. 
Numerous case studies on digital puppetry systems focus on puppetry performances (which the author will not review one by one here). The creation of digital and physical puppets and puppetry roles has given users greater autonomy and the capacity to exercise their creativity (such as learning about the characters or becoming familiar with puppetry performance). Users have the opportunity to collaborate in order to experience different puppetry roles. Based on the analysis of these studies, the author summarised four features of current digital puppetry performance: (1) oversimplified performances; (2) simplified puppetry characters; (2) simplified gestures; and (4) cultural significance.

Oversimplified performances. The puppetry performances of these studies have been excessively simplified by movement-capturing and animation rendering. For instance, the study on Cutout Animation reported limitations of the digital equipment [25], whereby the system was unable to capture all puppetry gestures and fast movements, as Barnes and colleagues mentioned: Unfortunately, our system is not capable of handling every puppet or action a user may wish to animate...puppets cannot be moved too quickly, as the Kanade-Lucas-Tomasi (KLT) algorithm assumes that the optical flow between frames is well approximated by small displacements without rotation. Users do not have the chance to experience historic puppetry performances, which include complex gestures. Traditional Chinese puppetry encompasses extensive operabased gestures. The current digital technology could mislead users to have an over simplified understanding of puppetry performances.

Simplified puppetry characters. In the third case study of Shadow Story [26], the interactive interface of the puppetry characters - which simplified the characters of traditional shadow puppetry - only offered a simple digital pen and basic colours for users to create the characters. Fig.1 shows that it is not difficult to see that the users' characters lacked the basic elements of traditional puppetry. Thus, it is crucial to provide users with a more accurate understanding of the characters. Developing ways of using digital technology to enhance the creation of shadow puppets could be a direction for future design. 


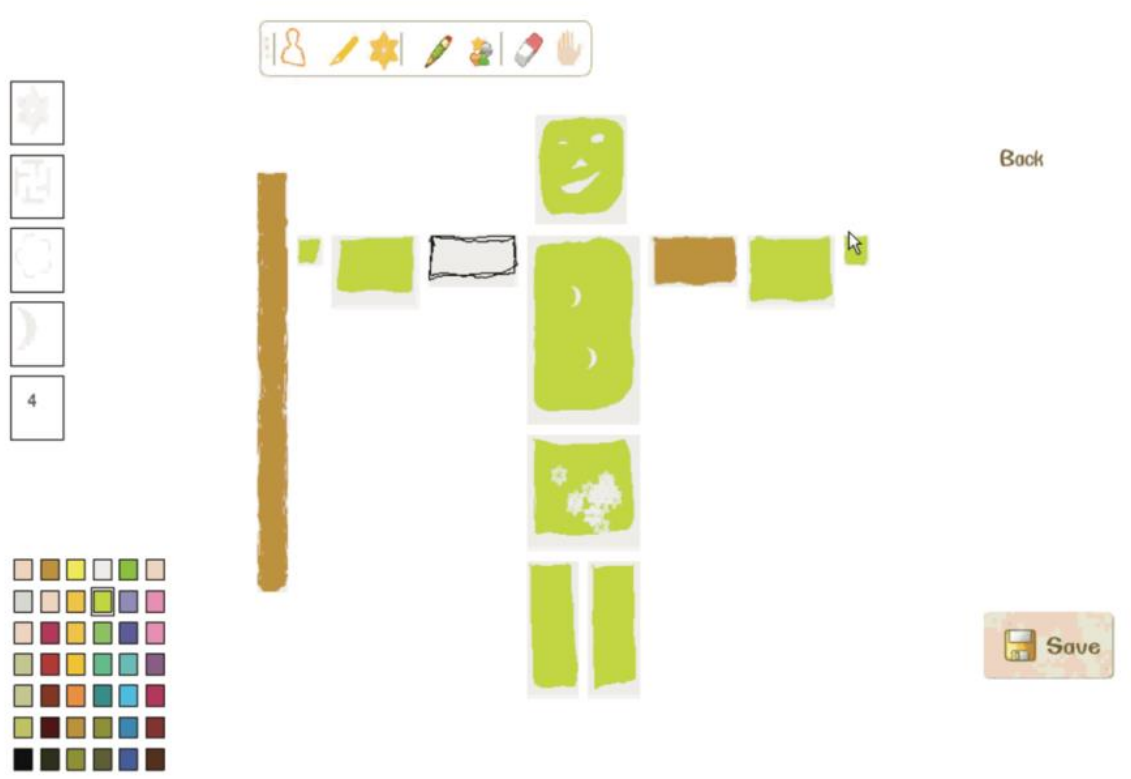

Fig. 1. Interface of the 'design' mode for creating characters (Lu et al. 2011).

Simplified gestures. The gestural performance was missed or simplified by the running of the digital system [27]. The system interactions in user engagements and the actual operation of the puppets have visible distinctions. Prior research has not deeply explored the potential for helping viewers to engage with traditional puppetry performance. For instance, the Shadow Story system offers users handheld sensors to conduct gestural interactions, rather than letting them have physical interactions with shadow puppets. Although Wan and colleagues exploited a Kinect-based system to support users in personally manipulating shadow characters with their gestures [28], their gestures still differed greatly from the gestures of traditional shadow play. Furthermore, Liang and colleagues developed a hand gesture-based interaction and animation data repository to generate interactive animation using shadow play [29]. However, the gestures were only classified by the roles of the shadow play, and the users did not have chance to take part in real shadow play. They did not aspire to this, and the gesture-based interactions were simplified to fit with the game experience.

Cultural significance. Lastly, cultural appreciation (e.g. the cultural meaning of gestures and stories) of puppetry is not well supported, as most prior research is entertainment-oriented. Current digital systems do not portray traditional Chinese puppetry as part of ICH [5] in the sense of helping users to learn about the relevant history and customs by experiencing a digital puppetry performance. However, Lu and colleagues tried to work with professional puppeteers of traditional Chinese shadow puppetry in order to identify the characters as elements to be used in a digital system. This collaboration resulted in a series of traditional cultures as available design elements for the digital system; the present elements are only associated with puppetry 
characters, but for the puppetry gestures, no relevant identification was carried out. Thus, conducting fieldwork with professional puppeteers to gather more elements from traditional puppetry (e.g. puppetry gestures) could provide a cultural reference for the design phase of this the future study.

\subsection{Interactive Technology for Puppetry Appreciation}

This section focuses on some interactive digital systems intended to support the appreciation and understanding of puppetry. Current research has mainly adopted emotional expression/involvement and storytelling as two different methods to facilitate understanding and appreciation.

Emotional expression. As early as 1978, Dolby mentioned that audiences' emotional expression acting as one of the main entry points, could be used to improve their appreciation of traditional Chinese puppetry [30]). Bai and colleagues designed an interactive prototype called Fing Augmented Reality (AR), which aimed to enhance children's complex cognitive and social development. Through a series of puppetry games, children acted out their emotions, beliefs and desires, and interpreted the roles based on their experience with the game. In this project, AR was exploited to help children understand the emotions of the puppetry characters during the game. Another, similar project is called emoPuppet; it is an interactive digital-physical puppet that helps children to express and understand emotions [31]. Based on a smartphone application, children observed and appreciated simple puppetry characters to learn about facial expressions. They were engaged effectively in the experience and developed a basic understanding of puppetry, alongside the interactive technology that also improved their creativity and social skills. Shi and colleagues combined characteristics of Chinese folk culture to design a digital shadow puppet, and developed an interactive system that exploited Kinect-based interactions and sensors to capture children's movements, the goal being to help them express their emotions with shadow puppets [32]. Their assessment showed that the system helps children develop a fundamental understanding of shadow puppetry.

However, emotional expressions and experiences did not integrate the features of puppetry characters or specific classical stories in these aforementioned case studies. In other words, users' expressional emotions were disjointed from the puppetry itself; the characters and enjoyment were not embodied. Therefore, the oversimplified gestures and basic emotional expressions seem to have limited the cultural appreciation of puppetry shows.

A potential direction for design is how to systematically display highly complex gestures in the system to create a richer experience for users. In addition, while using emotions to enhance children's puppetry appreciation is worthwhile, a large number of audiences of traditional Chinese puppet shows are adults. Hence, there is an opportunity to explore how puppet gestures support deeper cultural appreciation among adult audiences. Traditional Chinese puppetry is a sort of sacrificial activity that originated 
in Chinese agricultural society [3], most Chinese puppetry describes folktales. Therefore, the author believes that it is necessary to explore how to use emotional expressions to engage these audiences in order to help them appreciate the cultural significance of traditional Chinese puppetry.

Digital storytelling. Some research has focused more on using storytelling with digital animation to boost appreciation of traditional puppetry. For instance, based on the photon mapping method [33], Zhu and colleagues developed a prototype that turned traditional shadow plays into electronic forms that provided the audience with immersive storytelling [34]. They offered delicate lighting effects and realistic martial arts sequences to reproduce the classical Chinese folk story The butterfly lovers. However, the digital animation only focused on reproduction, and the subtle meanings of the shadow play gestures were not well-explained. The background, dialogue and traditional music of this story were not expressed, lacking an interpretation to support the users' appreciation. Another shadow play animation system exploited the Rapidly exploring Random Trees (RRTs)-Connect algorithm to capture the characteristics of motion from traditional Chinese shadow play, and depicted the 'emulational' animation for audiences [35, 36]. The animation system recreates realistic shadow character movements to provide an aesthetically-pleasing environment. Although $\mathrm{Hsu}$ and $\mathrm{Li}$ further explored how to use delicate animation to develop an original story, their research only centred on delivering experiences to users with the same or similar cultural backgrounds, or those who already appreciated the aesthetics of puppetry.

Prior research has failed to investigate how to narrate traditional Chinese stories via shadow puppetry to audiences, especially audiences from different cultural backgrounds. Using interactive technology in storytelling to enhance the appreciation of cross-cultural audiences is a potential insight for design that needs to be explored [37]. Furthermore, the cultural relevance of puppetry narratives and the in-depth meaning of puppetry gestures need to be effectively interpreted for audiences [38, 39]. Although expressing emotions through interactive technology is a valuable way of increasing audiences' appreciation of puppetry, there are other important opportunities for interactive technology to help audiences bridge cultural differences that have not been deeply examined. These include digital systems that use gestures to enhance crosscultural audiences' appreciation of puppetry and its deeper relationship with Chinese culture.

\section{Discussion}

The author reviewed different research projects that used interactive technology to enhance the performance and appreciation of puppetry. Current studies have effectively supported users in experiencing puppetry briefly, and traditional puppetry has also been utilised to convey culture to engage users in game entertainment or social learning. How to engage audiences or viewers' interest in traditional Chinese puppetry is always a key aspect of safeguarding it [40]. However, through analysing the extensive case 
studies that integrate interactive technology, the author believed that the current case studies did not make a clear distinction of both learning and appreciation of traditional Chinese puppetry. Thus, indirectly leading to the audiences or viewers to not understand the cultural significance of traditional Chinese puppetry, and lose their interest in it. However, the following points need to be considered: (1) Traditional puppetry performances (e.g. gestures, movements) are oversimplified in interactive systems, which may lead users to have an incomplete understanding of traditional Chinese puppetry. Maintaining originality is necessary in the design phase. (2) In most of the case studies, children were the main target audience. Yet in traditional Chinese puppetry, a large number of audiences are adults. Thus, it is crucial to explore how to use interactive technology in order to design a way for adults to appreciate this art form. (3) Based on the findings from the point above, it is also necessary to determine ways to support adult audiences in grasping the cultural significance and folk customs of traditional Chinese puppetry. In integrating the interactive technology into the appreciation and experience of Chinese puppetry, it is extremely crucial to maintain the aesthetic and cultural significance in safeguarding Chinese puppetry. However, cultural significance is not embodied in current digital systems. (4) No particular case study has focused on supporting cross-cultural users in appreciating and experiencing traditional Chinese puppetry. The further study's main research goals is to investigate ways to use emotional expressions, digital storytelling and other methods in conjunction with interactive technology to help multi-cultural users comprehend traditional Chinese puppetry.

Furthermore, Determining the correct role and positioning of technology is a complicated and controversial topic, and puppetry stakeholders are often critical in questioning and problematising the status of technology [41]. Therefore, examining the possible relationships between traditional Chinese puppetry and interactive technology brings up questions as to whether digital puppetry performances may threaten traditional performance and skills or other intangible elements. Additional questions remain regarding whether changing audiences' perception of Chinese puppetry will potentially decrease audiences viewership at traditional theatres. The current study cannot determine whether interactive technology can ethically and reasonably be integrated into Chinese ICH; designers are currently exploring different approaches to this question. However, the findings suggest that shifting the emphasis of interactive technology in puppetry from entertainment to the support of audiences' appreciation and understanding would not threaten traditional performances. The future research should examine how interactive technology assists cross-cultural audiences in overcoming cultural barriers and further engages their interest.

\section{References}

1. Huang, Y., Lioret, A: Cerebral interaction and painting. In: Proceedings of the SIGGRAPH Asia 2013 art gallery, pp. 21. Hong Kong, China (2013).

2. Zhao, S., Kirk, D: Using Interactive Digital Media to Support Transcultural Understanding of Intangible Chinese Cultural Heritage. In: Proceedings of CHI 2016 Conference 
Workshop-Involving the CROWD in future MUSEUM experience design, San Jose, CA (2016).

3. Chen, FPL., Clark, B.: A survey of puppetry in China. Asian Theatre Journal 27(2), 333365 (2010).

4. Zhao, S., Kirk, D., Bowen, S., Wright, P.: Cross-cultural understanding of Chinese traditional puppetry: integrating digital technology to enhance audience engagement. International Journal of Intangible Heritage 14, 140-156 (2019).

5. Xu, ZM., Xin, XF.: The phylogeny of Chinese puppet show. Literature of Shandong Press, Shandong, China (2007).

6. Wu, Z.: The research of digitalized technology of the puppet show with the motion capture technology. Journal of University of Electronic Science and Technology of China, 6-7 (2009).

7. Fraser, M., Stanton, D., Ng, KH., Benford, S., O’Malley, C., Bowers, J., Taxén, G., Ferris, K., Hindmarsh, J.: Assembling history: Achieving coherent experiences with diverse technologies. In: Proceedings of the eighth European conference on computer supported cooperative work, pp.14-18. Helsinki, Finland (2003).

8. Kortbek, K., Grønbæk, K.: Communicating art through interactive technology: New approaches for interaction design in art museums. In: Proceedings of the 5th Nordic Conference on Human-Computer Interaction: Building Bridges, pp. 229-238. Lund, Sweden (2008).

9. Huang, C.H., Huang, Y.T.: An Annales School- based Serious Game Creation Framework for Taiwanese Indigenous Cultural Heritage. Journal on Computing and Cultural Heritage (JOCCH) 6(9), 9-31 (2013).

10. Candy, L., Ferguson, S.: Interactive Experience in the Digital Age: Evaluating New Art Practice, Springer Series on Cultural Computing, London, UK: Springer (2014).

11. Huang, Y.: Creation methodology of interactive art installation based on philosophyunderstanding projection: Recreation of traditional Chinese painting. In: Proceedings of the 2015 Virtual Reality International Conference, pp. 7. Laval, France (2015).

12. Chang, Y.H., Lin, Y.K., Fang, R.J., Lu, Y.T.: A Situated Cultural Festival Learning System Based on Motion Sensing. Eurasia Journal of Mathematics, Science \& Technology Education 13(3), 571-588 (2017).

13. Lombardo, V., Pizzo, A., Damiano, R.: Safeguarding and Accessing Drama as Intangible Cultural Heritage. Journal on Computing and Cultural Heritage (JOCCH), 9(1), 5 (2016).

14. Maye, LA., Bouchard, D., Avram, G., Ciolfi, L.: Supporting Cultural Heritage Professionals Adopting and Shaping Interactive Technologies in Museums. In: Proceedings of the 2017 Conference on Designing Interactive Systems, pp. 221-232. Edinburgh, United Kingdom (2017).

15. Meroni, A., Sangiorgi, D.: Design for services, Gower Publishing, Aldershot, UK (2011).

16. Lu, Z., Annett, M., Fan, M., Wigdor, D.: "I feel it is my responsibility to stream": Streaming and engaging with intangible cultural heritage through livestreaming. In: Proceedings of the SIGCHI conference on human factors in computing systems, pp. 229. Glasgow, UK (2019).

17. Champion, E.: Cross-cultural learning, heritage, and digital games' in J. Hartley, W. and W. $\mathrm{Qu}$, eds., Reorientation: Trans-Cultural. Trans-Cultural, Trans-Lingual, Transmedia Studies in Narrative, Language, Identity and Knowledge, Shanghai: Fudan University Press, pp. 218-233 (2016).

18. Pujol, L., Champion, E.: Evaluating Presence in Cultural Heritage Projects', International Journal of Heritage Studies, 18, 83-102 (2012). 
19. Zhao, S., Kirk, D., Bowen, S., Wright, P.: Enhancing the Appreciation of Traditional Chinese Painting Using Interactive Technology. Multimodal Technologies and Interaction 2(2), 16 (2018).

20. Sørensen, S., Carman, J: Heritage Studies: Methods and Approaches, Routledge, Abingdon (2009).

21. Soderland, A.: The History of Heritage: A Method in Analysing Legislative Historiography. In: Heritage Studies: Methods and approaches, edited by Marie Louise Stig Sørensen, John Carman, 55-84. Routledge, Abingdon (2009).

22. Sturman, DJ: Computer puppetry. IEEE Computer Graphics and Applications 18(1), 38-45 (1998).

23. Shin, HJ., Lee, J., Shin, SY., Gleicher, M.: Computer puppetry: An importance-based approach. ACM Transactions on Graphics 20(2), 67-94 (2010).

24. Marshall, P., Rogers, Y., Scaife, M.: Puppet: A virtual environment for children to act and direct interactive narratives. In: 2 nd international workshop on narrative and interactive learning environments, pp. 8-15 (2002).

25. Barnes, C., Jacobs, DE., Sanders, J., Goldman, DB., Rusinkiewicz, S., Finkelstein, A., Agrawala, M.: Video puppetry: A performative interface for cutout animation. ACM Transactions on Graphics 27(5), 124 (2008).

26. Lu, F., Tian, F., Jiang, Y., Cao, X., Luo, W., Li, G., Zhang, X., Dai, G., Wang, H.: Shadow Story: Creative and collaborative digital storytelling inspired by cultural heritage. In: Proceedings of the SIGCHI conference on human factors in computing systems (CHI '11), pp. 1919-1928. Vancouver, BC, Canada (2011).

27. Zhao, S.: Exploring How Interactive Technology Enhances Gesture-Based Expression and Engagement: A Design Study. Journal of Multimodal Technologies Interact 3(1), (2019).

28. Wan, B., Wen, XJ., An, L., Ding, X.: Interactive shadow play animation system. International Journal of Computer, Electrical, Automation, Control and Information Engineering 9(1), 127-132 (2015).

29. Liang, H., Deng, S., Chang, J., Zhang, JJ., Chen, C., Tong, R. 2017.: Semantic framework for interactive animation generation and its application in virtual shadow play performance. Virtual Reality 22(2), 149-165 (2017).

30. Dolby, W.: The origins of Chinese puppetry. Bulletin of the School of Oriental and African Studies, University of London 41(1), 97 -120 (1978).

31. Martínez, JI.: emoPuppet: Low-cost interactive digital-physical puppets with emotional expression. In: Proceeding of the 11th conference on advances in computer entertainment technology (ACE '14), Article No. 44. ACM, New York, NY (2014).

32. Shi, Y., Ying, F., Chen, X., Pan, Z., Yu, J.: Restoration of traditional Chinese shadow playPiying art from tangible interaction. Journal of Visualization and Computer Animation 25(1), 33-43 (2013).

33. Jensen, HW.: Realistic image synthesis using photon mapping, AK Peters, Natick, MA (2001).

34. Zhu, Y-B., Li, C-J., Shen, IF., Ma, K-L., Stompel, A.: A new form of traditional art: Visual simulation of Chinese shadow play. In: Proceedings of ACM SIGGRAPH 2003 Sketches \& Applications (SIGGRAPH '03), New York, NY (2003).

35. Hsu, S-W., Li, T-Y.: Planning character motions for shadow play animations. In: Proceedings of computer animation and social agents 5, 184-190 (2005).

36. Hsu, S-W., Li, T-Y.: Generating secondary motions in shadow play animations with motion planning techniques. In: Proceedings of SIGGRAPH 2005 Conference on Sketches, Article No. 69. Los Angeles, California (2005). 
37. Hickey, MG.: Asian Indian celebrations of ethnicity: Perspectives from the mid-western United States. International Journal of Intangible Heritage 7, 31-44 (2012).

38. Giaccardi, E.: Things we value. In Magazine Interactions 18(1), 17-21 (2011).

39. Hawkins, T., Cohen, J., Debevec, P.: A photometric approach to digitizing cultural artifacts. In: Proceedings of the 2001 conference on virtual reality, archeology, and cultural heritage (VAST '01), pp. 333-342. ACM, New York, NY (2011).

40. Zhao, S.: An Analysis of Interactive Technology's Effect on the Appreciation of Traditional Chinese Painting: A Review of Case Studies. The International Journal of New Media, Technology and the Arts, 14 (3), 1-12 (2019).

41. Lawson, S., Kirman, B., Linehan, C., Feltwell T. and Hopkins, L.: Problematising upstream technology through speculative design: The case of quantified cats and dogs. In: Proceedings of the 33rd annual ACM conference on human factors in computing systems, pp. 26632672. Seoul (2015). 